\title{
Experimental and numerical analysis for thermal-hydraulic characteristics in circular tubes with twisted tape and wire coil inserts
}

\author{
Shuai Wang*, Kai Wang, Kun Lu and Qingguo Zhang \\ Huadian Electric Power Research Institute Co., Ltd., Hangzhou, Zhejiang, 310030, China
}

\begin{abstract}
Thermal-hydraulic characteristics in circular tubes with twisted tape ( $\mathrm{Y}=4.762,7.143$ and 9.524) and wire coil $(\mathrm{p}=20 \mathrm{~mm}, \mathrm{e}=2 \mathrm{~mm})$ inserts were studied experimentally and numerically. It was found from experimental analysis that Nusselt number and friction factor in tubes with twisted tape inserts were 38\%$57 \%$ and $148 \%-202 \%$, respectively, higher than those of plain tubes. When wire coil was inserted in plain tubes, the Nusselt number and friction factor grew by $75 \%-94 \%$ and $685 \%-792 \%$, respectively. In particular, Nusselt number and friction factor in tubes with twisted tape and wire coil inserts were $113 \%-139 \%$ and $836 \%-874 \%$, respectively, greater than those of plain tubes. Furthermore, PEC value of tubes with the twisted tape and wire coil inserts was larger than all others. Numerical analysis shows that a large tangential velocity can be generated after inserting a twisted band or coil in the tube. The angle between the temperature gradient vector and velocity vector was significantly less than $90^{\circ}$. So collaborative degree between temperature field and velocity field was improved.
\end{abstract}

\section{Introduction}

Heat transfer enhancement technology with the inserts inside tubes does not change the shape of heat transfer surface. Moreover, the processing is simple without replacing significant features of shell and tube heat exchangers. So it has been widely applied to the old factory. S.K. Saha and A. Dutta [1] investigated heat transfer and pressure drop characteristics in a circular tube fitted with regularly spaced twisted-tape elements. It is observed that reducing tape-widths yields poor results and higher-than-zero phase angle is of no use. S. Fahed and L.M. Chamra [2] published pressure drop and heat transfer data for both micro fin tube and twistedtape inserts in laminar flow. The results demonstrate that the twisted-tape gives better heat transfer enhancement with reduced twist ratio. E. Smith [3] provided experimental data of heat transfer and flow friction in a circular tube fitted with regularly spaced twisted tape elements. Their studies suggest the larger twist ratio is, the bigger heat transfer coefficient becomes. P. Promvonge and S. Eiamsa-ard [4] reported heat transfer behaviors in a tube with combined conical-ring and twisted-tape insert. The experimental results reveal that the tube fitted with the conical-ring and twisted-tape provides Nusselt number and enhancement efficiency are $4 \%-10 \%$ and of $4 \%-8 \%$, respectively, higher than that with the conical-ring alone. S. W. Chang [5] tested heat transfer and pressure drop in tube with broken twisted tape insert. It is found that heat transfer coefficients and friction factors in the tube fitted with the broken twisted tape are 1.28-2.4 and 2-4.7 times of those in the tube fitted with the smooth twisted tape, respectively. The experimental study on three wire coils of different pitches inserted in a smooth tube was carried out by A. Garcia [6]. Their studies reveal wire coils remarkably increase heat transfer with Reynolds numbers ranging from 200 to 1000 . A.W. Date [7] presented numerical prediction of laminar flow and heat transfer in a tube fitted with twisted tape swirl generator. In their work, the influences of property variations and buoyancy on system calculations are also determined. Numerical and experimental analyses were carried out to study thermalhydraulic characteristics of air flow inside a circular tube with different tube inserts by Y.W. Chiu and J.Y. Jang [8]. It is found that heat transfer coefficient and pressure drop in tubes with the longitudinal strip inserts (without hole) were $7-16 \%$ and $100-170 \%$, respectively, greater than those of plain tubes. S. Eiamsa-ard [9] provided simulation data of swirling flow and convective heat transfer in a circular tube induced by means of loose-fit twisted tapes. It is visible that twisted tape inserts for $\mathrm{y} / \mathrm{w}=2.5$ with $\mathrm{CR}=0.0,0.1,0.2$ and 0.3 can enhance heat transfer rates up to $73.6 \%, 46.6 \%, 17.5 \%$ and $20 \%$, respectively, in comparison with those of the plain tube.

Above-mentioned literature review demonstrates the extensively experimental or numerical analysis for thermal-hydraulic characteristics in circular tubes with twisted tape or wire coil inserts. However, so far, experimental or numerical analysis of circular tubes with twisted tape or wire coil inserts has not applied to actual production very well. Hence, the aim of the current study is to reveal the internal mechanism of heat transfer

\footnotetext{
* Corresponding author: shuai-wang@chder.com
} 
enhancement in tubes with different inserts by numerical simulation. Then, structure optimization is carried out with experimental data to obtain the optimal inserts inside tubes. Finally, it can be applied to actual production with providing a reliable basis for the design of heat exchangers.

\section{Experimental set-up and calculation model}

Schematic diagram of experiment apparatus is shown in Fig.1. The working medium in the experiment was air. The test loop consists of a test section, hot air loop, cold air loop and data acquisition system. The test section was a horizontal concentric tube heat exchanger. The inner and outer tubes $\left(d_{\text {in }}=25 \mathrm{~mm}, d_{\text {out }}=76 \mathrm{~mm}\right)$ were made from red copper and stainless steel, respectively. Except for the loop component, a full set of instrument for measuring the temperature and flow rate of fluid was installed at the important points in the circuit. The ambient air was pumped by the air compressor and passed through flow control valve, glass rotameter, electrical heater, the stable period $(500 \mathrm{~mm})$ and test section $(2000 \mathrm{~mm})$ in turn. The hot air and cold air temperatures at the inlet and outlet were measured by copper-constantan thermocouple. Meanwhile, U tube manometer was employed to measure the pressure drop across the test section. Finally, the inlet stable section, heat section and test section were insulated by an elastomeric thermal insulation of which thermal conductivity was $0.03 \mathrm{~W} /(\mathrm{m} \cdot \mathrm{K})$ to minimize heat loses.

Fig. 2 presents a sketch of twisted tape inserted in a tube, where $\mathrm{H}$ stands for axial distance, $\mathrm{B}$ stands for tape width $(\mathrm{B}=20 \mathrm{~mm})$ and di stands for pipe diameter $\left(d_{\mathrm{i}}=21\right.$ $\mathrm{mm})$. The twisted tapes of different dimensionless distance $\left(\mathrm{Y}=\mathrm{H} / d_{\mathrm{i}}=4.762,7.143\right.$ and 9.524) were inserted in plain tubes in the experiment. Fig. 3 demonstrates a sketch of wire coil inserted in a tube, where $\mathrm{p}$ stands for helical pitch and e for wire diameter. The geometrical parameters of wire coil in the experiment are p $(20 \mathrm{~mm})$ and e $(2 \mathrm{~mm})$.

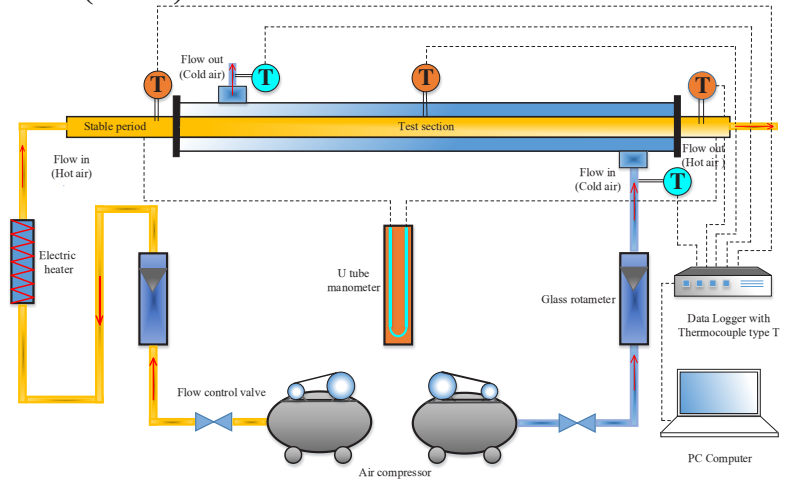

Fig.1. Schematic diagram of experiment apparatus

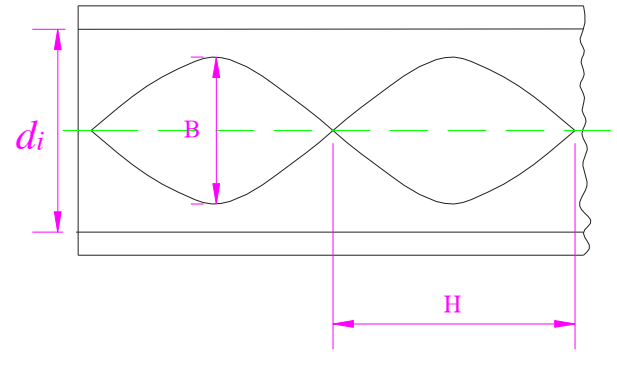

Fig.2. Geometric structure of a twisted tape

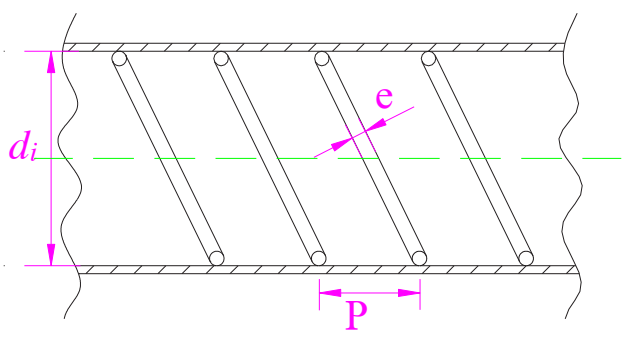

Fig.3. Geometric structure of a wire coil

\section{Numerical method}

For the turbulent fluid flow, k- $\omega$ model was chosen. The flow and pressure equations were solved with SIMPLEC algorithm, which is one of the three widely used velocity-pressure coupling algorithm. The Second Order Upwind algorithm was employed in the discretization of the viscous and source terms. Because heat transfer was included, the energy equation was set. The energy equation residuals were taken as $1 \mathrm{e}-6$, other variables taken as 1e-3. For the boundary conditions, Velocityinlet (velocity inlet) and Outflow (free flow outlet) were selected. The wall temperature of tube was set to a constant temperature. In addition, twisted tape and wire coil were considered as insulation, which might lead to some errors.

Since the ratio of tube length and diameter was 100:1 in the simulation, velocity, temperature and pressure distribution along the longitudinal direction of tubes were scaled down, which might cause distortion of the visual.

\section{Data reduction}

The data reduction of the measured results was summarized in the following procedures. For fluid flows in a concentric tube heat exchanger, the heat transfer rate of cold air in the test section could be expressed as

$$
Q_{\mathrm{c}}=m_{\mathrm{c}} C_{\mathrm{p}, \mathrm{c}}\left(T_{\mathrm{c}, \text { out }}-T_{\mathrm{c}, \text { in }}\right)
$$

Where $m_{\mathrm{c}}$ was the mass flow rate of cold air, $C_{\mathrm{p}, \mathrm{c}}$ was the specific heat of cold air, $T_{\mathrm{c}, \text { out }}$ and $T_{\mathrm{c}, \text { in }}$ were the outlet and inlet cold air temperatures, respectively.

While the heat transfer rate of hot air was

$$
Q_{\mathrm{h}}=m_{\mathrm{h}} C_{\mathrm{p}, \mathrm{h}}\left(T_{\mathrm{h}, \text { in }}-T_{\mathrm{h}, \text { out }}\right)
$$

Where $m_{\mathrm{h}}$ was the hot air mass flow rate; $T_{\mathrm{h}, \text { out }}$ and $T_{\mathrm{h}, \text { in }}$ were the outlet and inlet hot air temperatures, respectively. 
The heat transfer rate of red copper tube used in the calculation was determined as follows

$$
\begin{aligned}
& Q_{\mathrm{t}}=h_{\mathrm{i}} A\left(T_{\mathrm{wo}}-T_{\mathrm{m}}\right) \\
& T_{\mathrm{m}}=\left(T_{\mathrm{h}, \mathrm{in}}+T_{\mathrm{h}, \text { out }}\right) / 2 \\
& T_{\mathrm{wo}}=\sum T_{\mathrm{w}} / 10
\end{aligned}
$$

Where $A$ was the area of inner tube, $T_{\text {w }}$ was the local wall temperature. It was measured at the depth of $1.5 \mathrm{~mm}$ away from the outer surface. $T_{\mathrm{m}}$ was the average temperature of hot and cold air. Hence, the average heat transfer coefficient for the inner tube could be obtained as follows.

$$
h_{\mathrm{i}=} m_{\mathrm{h}} C_{\mathrm{p}, \mathrm{h}}\left(T_{\mathrm{h}, \mathrm{in}}-T_{\mathrm{h}, \text { out }}\right) / A\left(T_{\mathrm{wo}}-T_{\mathrm{m}}\right)
$$

The local Nusselt number of hot air was calculated by means of

$$
N u=h_{\mathrm{i}} d_{\mathrm{i}} / \lambda
$$

The Reynolds number was given by

$$
R e=\rho_{\mathrm{h}} u_{\mathrm{m}} d_{\mathrm{i}} / \mu
$$

Pressure drop tests were carried out under isothermal conditions. The inner diameter was used as the reference diameter to calculate all friction factors. Fanning coefficients were determined from mass flow rate and pressure drop by means of

$$
f=\Delta P /\left[\left(L / d_{\mathrm{i}}\right) \cdot\left(\rho_{\mathrm{h}} u_{\mathrm{m}}^{2} / 2\right)\right]
$$

The performance evaluation for heat transfer enhancement was calculated by means of

$$
P E C=\left(N u / N u_{0}\right) \cdot\left(f / f_{0}\right)^{-1 / 3}
$$

\section{Results and discussion}

Before performing experiments over the twisted tape or wire coil, the plain tube was tested in order to adjust the experimental setup and check its reliability. Fig.5 and Fig.6 demonstrated that Nusselt number of experiment agreed well with Dittus-Boelter equation whose errors were $\pm 3-8 \%$, while Friction factor of experiment agreed well with Petukhov equation whose errors were $\pm 2-6 \%$. Therefore, the experimental system had higher reliability and measuring precision. It was also observed that the errors between the simulation results with standard $\mathrm{k}-\omega$ model and experimental results were within $5 \%$. Therefore, the standard $\mathrm{k}-\omega$ model was used to perform three-dimensional numerical simulation on heat transfer and resistance of tubes with twisted tape and wire coil inserts.

Fig.7 and Fig. 8 illustrated simulation and experiment results of $\mathrm{Nu}$ and $\mathrm{f}$ for different objects inserted into tubes, respectively. It was observed that the errors between the simulation results with standard k- $\omega$ model and experimental results were within $6 \%$. In terms of $\mathrm{Nu}$, simulation results were larger than experiment results when $\mathrm{Re}$ is smaller than 12000 , while simulation results were smaller than experiment ones when Re was larger than 12000. In terms of $f$, this phenomenon could not be obtained.

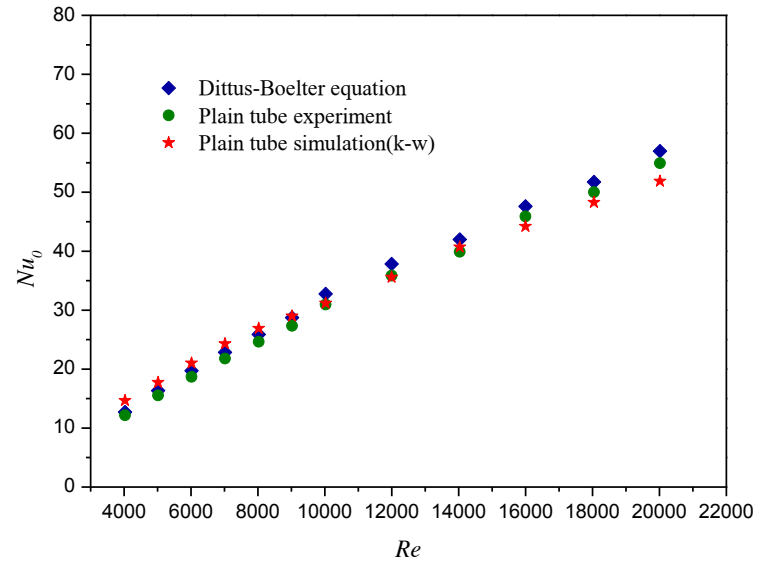

Fig.5. Simulation and experiment results of $N u_{0}$ in the plain tube

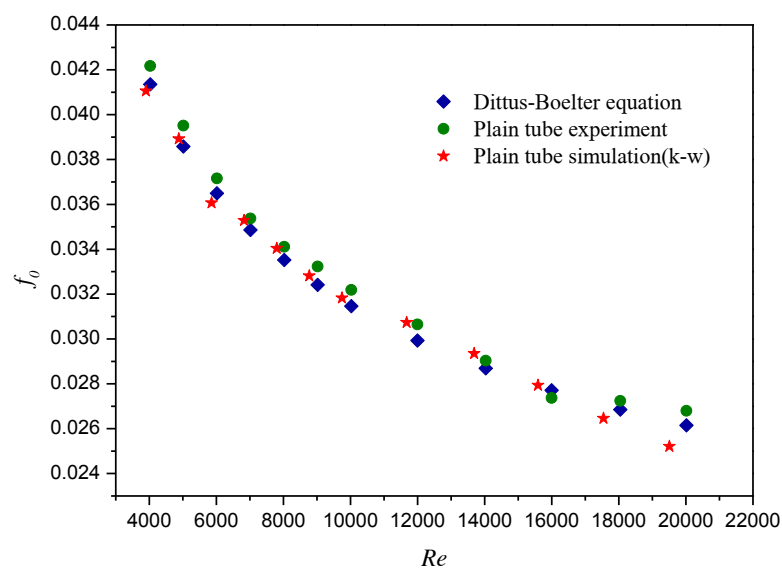

Fig.6. Simulation and experiment results of $f_{0}$ in the plain tube

As is expected, Nusselt number increased and the friction factor decreased as the Reynolds number increased. Furthermore, Nusselt number and friction factor of tubes with twisted tape or wire coil inserts were significantly higher than those of plain tubes. When twisted tape or wire coil was inserted in tubes, the laminar boundary layer near the wall was damaged with continuously mixing of fluid in the laminar boundary layer and the core area. Thus, heat transfer and resistance were enhanced. In terms of tubes with twisted tape inserts, however, the smaller the dimensionless distance was, the better the heat transfer performance was and the larger the friction factor was. The reason was that mixing of fluid in the laminar boundary layer and the core area was more sufficient, which led to larger heat transfer and resistance.

Specifically, Nusselt number and friction factor in tubes with twisted tape inserts were $38 \%-57 \%$ and $148 \%-202 \%$, respectively, higher than those of plain tubes. When wire coil was inserted in plain tubes, the Nusselt number and friction factor grew by $75 \%-94 \%$ and $685 \%-792 \%$, respectively. In particular, Nusselt number and friction factor in tubes with twisted tape and wire coil inserts were $113 \%-139 \%$ and $836 \%-874 \%$, respectively, greater than those of plain tubes. 
It could be clearly seen from Fig.7 and Fig.8 that the bigger the Nusselt number was, the larger the friction factor tended to be. The best goal is that Nusselt number of a tube is bigger but its friction factor is smaller. But the reality is just the opposite. Therefore, based on the research achievements of Webb et al. [10], PEC value was used to measure the comprehensive performance of heat transfer enhancement. Fig.9 demonstrated the variation of $P E C$ value with Reynolds number in terms of experiment data. It could be clearly seen that PEC value in tubes with twisted tape $(Y=4.762)$ inserts was the largest of all tubes with twisted tape inserts, larger than that of tubes with wire coil inserts. However, PEC value of tubes with the twisted tape and wire coil inserts was larger than all others, so it could be applied to the industry very well.

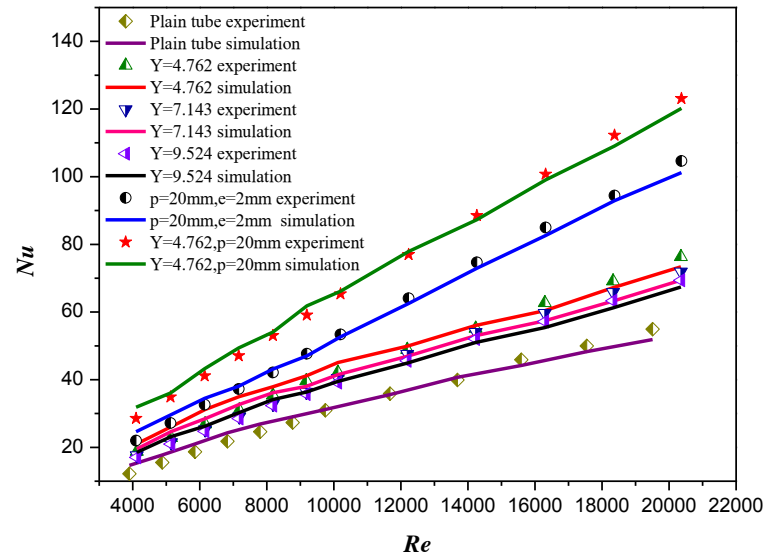

Fig.7. Simulation and experiment results of $N u$ for different objects inserted into tubes

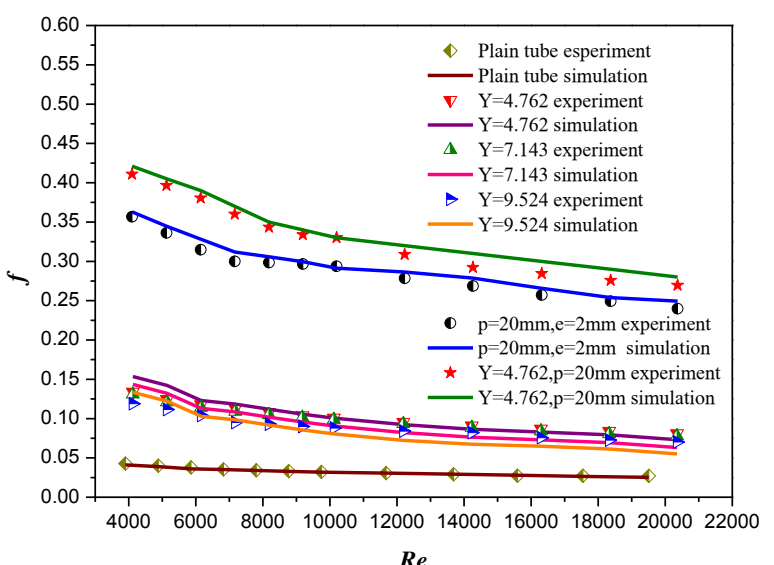

Fig.8. Simulation and experiment results of $f$ for different objects inserted into tubes

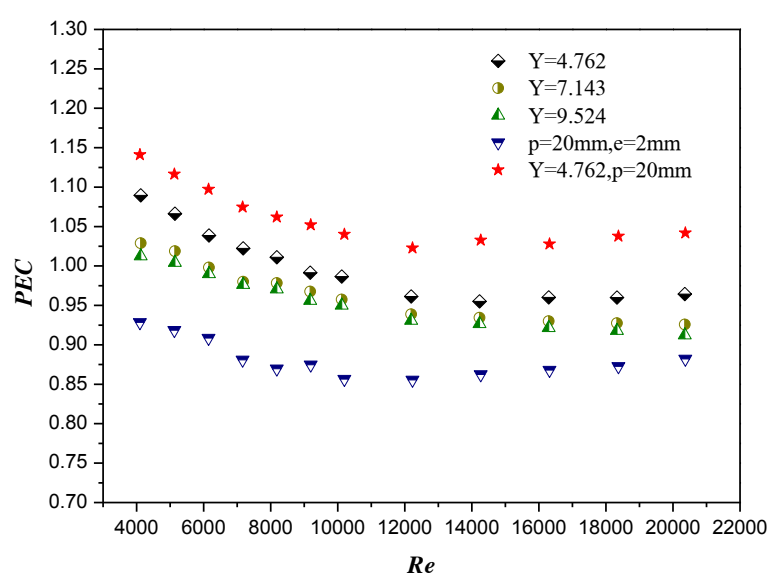

Fig.9. Variation of $P E C$ value with Reynolds number

Fig. 10 presents local velocity vector distribution of fluid in different tubes. It can be clearly seen that the shear velocity in plain tubes was very small compared to the axial velocity, which led to poor heat transfer. With respect to twisted tape inserts, a large tangential velocity of fluid was created. The tangential velocity created a centrifugal force that acted on fluid, which promoted the turbulent fluctuation of fluid, and then turbulent thermal resistance of the laminar boundary layer was reduced. In terms of wire coil inserts, wire coil led to separation of fluid boundary layer at the wall of inner tube, resulting in a great tangential velocity.

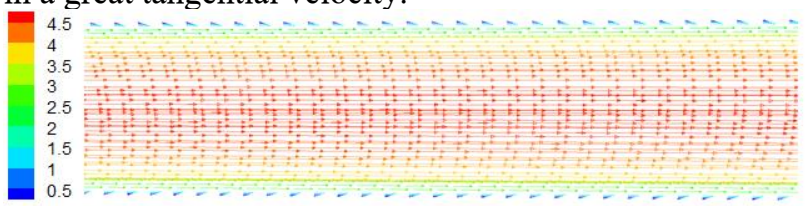

(a) Plain tubes
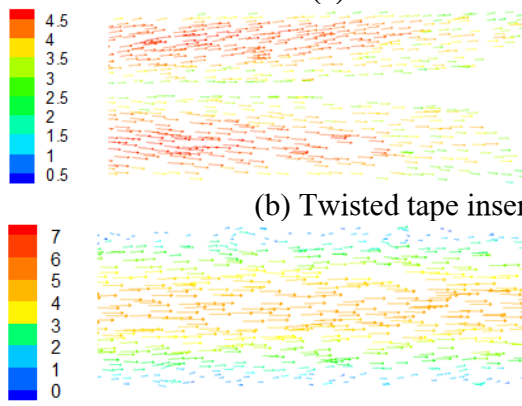

(b) Twisted tape inserts

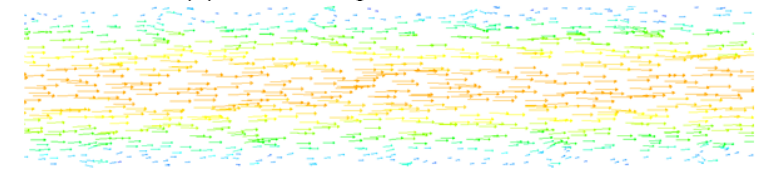

(c) Wire coil inserts

Fig.10. Local velocity vector distribution of fluid in different tubes

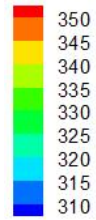

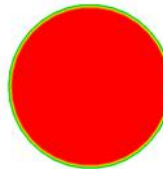

$z=0$

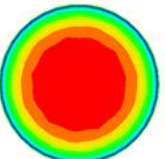

$z=0.1$

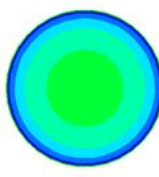

$z=1$

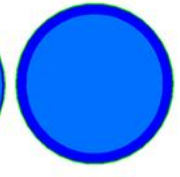

$z=2$ (a) Plain tubes 

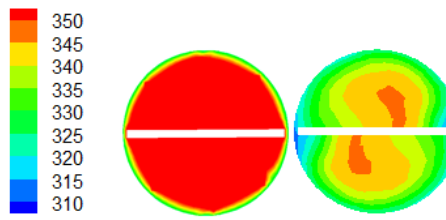

$z=0.1$
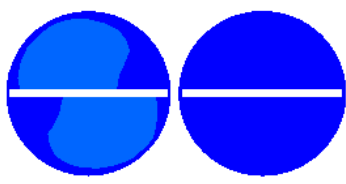

(b) Twisted tape inserts
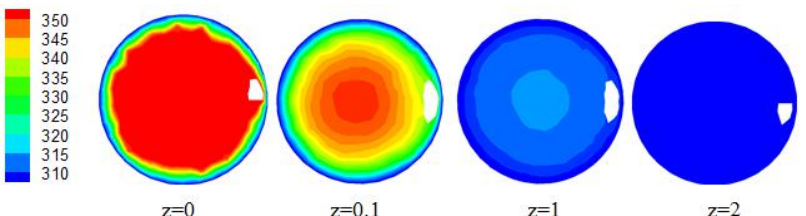

(c) Wire coil inserts

Fig.11. Temperature distribution of different cross-sections in different tubes

Fig.11 demonstrates the temperature distribution of different cross-sections in different tubes. The temperature distribution followed concentric circles in tubes with wire coil inserts. Specifically, the inner temperature was high, while the outer was low. As for tubes with twisted tape inserts, fluid in different crosssections was divided into two shares and their temperatures turned into apparent stratification before reaching fully-developed heat transfer. Moreover, they were centrosymmetric.

In terms of plain tubes, the temperature gradient of fluid at the laminar bottom was large, substantially perpendicular to the wall, while the velocity vector was mainly along the axis direction. In other words, the angle between the velocity vector and temperature gradient vector approached $90^{\circ}$. According to the field synergy theory of the academician named Guo Zengyuan, the synergies between the temperature gradient vector and velocity vector were poor. So the convective source term in energy equation was approximately zero, that is to say, the main heat transfer mode of fluid at the laminar bottom layer was thermal conductivity, which limited convective heat transfer between fluid and the tube wall.

After twisted tape or wire coil was inserted in the tube, tangential velocity was created and the velocity vector deviated from the axial direction. That is to say, the angle between the temperature gradient vector and velocity vector was significantly less than 90 . So collaborative degree between temperature field and velocity field was improved. In addition, the convective source term in energy equation was bigger than zero. Hence heat transfer enhancement was achieved.

\section{Conclusion}

1) When Reynolds number ranged from 3000 to 21000 , Nusselt number and friction factor in tubes with twisted tape inserts were $38 \%-57 \%$ and $148 \%-202 \%$, respectively, higher than those of plain tubes. When wire coil was inserted in plain tubes, the Nusselt number and friction factor grew by $75 \%-94 \%$ and $685 \%-792 \%$, respectively. In particular, Nusselt number and friction factor in tubes with twisted tape and wire coil inserts were $113 \%-139 \%$ and $836 \%-874 \%$, respectively, greater than those of plain tubes.

2) $P E C$ value in tubes with twisted tape $(Y=4.762)$ inserts was the largest of all tubes with twisted tape inserts, larger than that of tubes with wire coil inserts. However, $P E C$ value of tubes with the twisted tape and wire coil inserts was larger than all others, so it could be applied to the industry very well.

3) After twisted tape or wire coil was inserted in the tube, a great tangential velocity was created. The angle between the temperature gradient vector and velocity vector was significantly less than $90^{\circ}$. So collaborative degree between temperature field and velocity field was improved. Hence heat transfer enhancement was achieved.

\section{References}

1. S.K. Saha, A. Dutta, S.K. Dhal, INT J HEAT MASS TRAN, 44 (2001)

2. S. Fahed, L.M. Chamra, W. Chakroun, EXP THERM FLUID SCI , 18 (1999)

3. E. Smith, T. Chinaruk, P. Pongjet, INT COMMUN HEAT MASS TRANS, 33 (2006)

4. P. Promvonge, S. Eiamsa-ard, INT COMMUN HEAT MASS TRANS, 34 (2007)

5. S.W. Chang, T. L. Yang, J. S. Liou, EXP THERM FLUID SCI, 32 (2007)

6. A. Garcia, J. P. Solano, INT J HEAT MASS TRAN, 50 (2007)

7. A.W. Date, J ENHANC HEAT TRANSF,7 (2000)

8. Y.W. Chiu, J.Y. Jang, APPL THERM ENG, 29 (2009)

9. S. Eiamsa-ard, K.Wongcharee, S. Sripattanapipat, INT COMMUN HEAT MASS TRANS, 36 (2009)

10. R.L.Webb, Heat Mass Transfer, 24 (1981) 